\title{
Complejos ventriculares prematuros frecuentes luego de ablación de fibrilación auricular y su relación con el tono autonómico medido por parámetros del Holter ${ }^{\text {is }}$
}

\author{
Jorge A. Arroyave, Julián M. Aristizábal, Juan C. Díaz-Martínez, \\ Jorge E. Velásquez-Vélez, Jorge E. Marín-Velásquez, William Uribe y Mauricio Duque*
}

CES Cardiología - Universidad CES, Medellín, Colombia

Recibido el 12 de abril de 2016; aceptado el 2 de enero de 2017

Disponible en Internet el 2 de marzo de 2017

\section{PALABRAS CLAVE \\ Fibrilación auricular; Sistema nervioso autónomo; \\ Taquiarritmia; Ablación con catéter}

\begin{abstract}
Resumen
Introducción: La ablación con catéter de radiofrecuencia es un tratamiento prometedor para la fibrilación auricular. Pese a su seguridad, se ha evidenciado incremento en la frecuencia de complejos ventriculares prematuros y taquicardia ventricular no sostenida, asociados tal vez con influencias autonómicas.

Objetivo: Analizar la actividad autonómica mediante parámetros de monitorización Holter en pacientes llevados a esta intervención.

Metodología: Análisis retrospectivo de pacientes con complejos ventriculares prematuros frecuentes luego de aislamiento eléctrico de venas pulmonares por fibrilación auricular, seguidos mediante monitorización Holter entre octubre de 2010 y noviembre de 2014, a fin de comparar parámetros de variabilidad de la frecuencia cardiaca.

Resultados: $12,4 \%$ tuvo un incremento significativo del automatismo ventricular. Los datos demográficos en los grupos comparados no demostraron diferencias estadísticamente significativas. El promedio de complejos ventriculares prematuros durante las 24 horas fue de 5.363 con 237 por hora. En el grupo de estudio, $65 \%$ presentó taquicardia ventricular no sostenida. La variabilidad de la frecuencia cardiaca fue menor en los pacientes del grupo control $(p=0,000)$. La medida por frecuencias espectrales, evidenció una disminución en la frecuencia LF y HF para ambos grupos, con un valor menor para el grupo control $(p=0,000)$. La relación LF/HF para ambos grupos fue elevada $(p=0,612)$.

Conclusión: La ablación de venas pulmonares en el tratamiento de la fibrilación auricular puede conducir al desarrollo de complejos ventriculares prematuros frecuentes y taquicardia
\end{abstract}

\footnotetext{
Siguiendo la política de publicación de la revista, este artículo, en el que ha participado como coautor un miembro del Comité editorial, ha superado un estricto proceso de revisión por pares doble ciego y los autores no han tenido ninguna injerencia en la aprobación para su publicación.

* Autor para correspondencia.

Correo electrónico: mauricioduquemd@gmail.com (M. Duque).
} 
ventricular. El impacto sobre el sistema parasimpático y simpático puede ser un factor que precipite esta condición.

(C) 2017 Sociedad Colombiana de Cardiología y Cirugía Cardiovascular. Publicado por Elsevier España, S.L.U. Este es un artículo Open Access bajo la licencia CC BY-NC-ND (http:// creativecommons.org/licenses/by-nc-nd/4.0/).

\section{KEYWORDS}

Atrial fibrillation; Autonomic nervous system; Tachyarrhythmia; Catheter ablation
Frequent premature ventricular complexes following ablation of atrial fibrillation and their relationship to the autonomic tone measured with Holter parametres

\begin{abstract}
Introduction: Radiofrequency catheter ablation is a promising treatment for atrial fibrillation. Despite its security, and increase in the frequency of premature ventricular complexes and nonsustained ventricular tachycardia has been evidenced, which could have autonomic influences. Motivation: To analyse autonomic activity using Holter monitoring parametres in patients who underwent this procedure.

Methods: Retrospective analysis of patients with frequent premature ventricular complexes following electric isolation of pulmonary veins due to atrial fibrillation, followed up with Holter monitorisation between October 2010 and November 2014 with the goal of comparing heart rate variability parametres.

Results: $12.4 \%$ had a significant increase of ventricular automacity. Demographic data in compared groups did not reveal statistically significant differences. The average of premature ventricular complexes within 24 hours was of 5,363, with 237 per hour. In the study group, $65 \%$ showed non-sustained ventricular tachycardia. Heart rate variability was lower in patients from the control group $(p=0.000)$. Evaluation of spectral frequency evidenced a decrease in LF and HF for both groups, with a lower value for the control group $(p=0.000)$. HF/LF relationship for both groups was high $(\mathrm{p}=0.612)$.

Conclusion: Ablation of pulmonary veins in the treatment of atrial fibrillation can lead to the development of frequent premature ventricular complexes and ventricular tachycardia. The impact on the parasympathetic and sympathetic system could be a factor that triggers this condition.

(c) 2017 Sociedad Colombiana de Cardiología y Cirugía Cardiovascular. Published by Elsevier España, S.L.U. This is an open access article under the CC BY-NC-ND license (http:// creativecommons.org/licenses/by-nc-nd/4.0/).
\end{abstract}

\section{Introducción}

La fibrilación auricular es la arritmia cardiaca sostenida más común en la práctica clínica, y conlleva implicaciones importantes en cuanto a morbilidad y mortalidad ${ }^{1}$. Resulta de la interacción entre disparadores que inician el estímulo eléctrico y un sustrato consistente en un tejido vulnerable ${ }^{2}$. La medicación antiarrítmica usualmente es inefectiva, de tal modo que la ablación con catéter ha evolucionado durante los últimos quince años como uno de los tratamientos más prometedores al eliminar directamente algunos factores precipitadores ${ }^{3,4}$. En algunos casos, la terapia con radiofrecuencia para el aislamiento eléctrico de las venas pulmonares, incluso ha mostrado llevar a una curación potencial $^{3}$. Pese a la seguridad de esta terapia, se desconocen los efectos sobre el sistema nervioso autónomo (SNA) que pueden llevar a la generación de trastornos arrítmicos adicionales. La ablación de los plexos ganglionares autonómicos durante el aislamiento de venas pulmonares ha evidenciado, de manera anecdótica, incrementar la frecuencia de complejos ventriculares prematuros (CVPS) y de taquicardia ventricular no sostenida (TVNS) asociados probablemente con influencias autonómicas ${ }^{5}$. Debido a la localización y al modo de distribución de las estructuras nerviosas cardiacas, existen limitaciones en la realización de la ablación con radiofrecuencia, de modo que elimine las fibras puramente simpáticas o parasimpáticas del $\mathrm{SNA}^{6}$, impidiendo la aparición posterior de este tipo de trastornos.

Las CVPS se han descrito hasta en el 1\% de población clínicamente normal cuando se detecta por electrocardiograma, pero hasta en el $75 \%$ en monitorización Holter ${ }^{7-9}$. En general, se asocian con factores de riesgo cardiovasculares y no tienen repercusiones importantes en población con corazón estructuralmente normal, aunque no ocurre lo mismo en aquellos con alteraciones estructurales. Se ha reportado disfunción del SNA como consecuencia de la ablación con catéter de radiofrecuencia en varias taquicardias supraventriculares ${ }^{10}$, incluyendo fibrilación auricular, lo que pudiera llevar a una mayor frecuencia de CVPS en esta población. El análisis de esta prueba no invasiva se ha convertido en una de las herramientas más importantes para la valoración de la función del SNA, con base en el concepto de que fluctuaciones rápidas pueden reflejar específicamente el balance simpático vagal. En la monitorización Holter es posible analizar la variabilidad de la frecuencia cardiaca 
mediante diferentes parámetros y mediciones, y por ende evaluar dicha influencia simpático-vagal.

En la actualidad son pocos los datos acerca del posible papel del SNA en el desarrollo frecuente de CVPS y TVNS en población llevada a ablación de fibrilación auricular. Según conocimiento de los autores de este artículo, se han adelantado pocos estudios en relación con la influencia del tono autonómico en el desarrollo de estos trastornos arrítmicos ventriculares.

El objetivo de este estudio es analizar la actividad del SNA mediante parámetros de la variabilidad de la frecuencia cardiaca tomados de la monitorización Holter de 24 horas, en pacientes con CVPS posterior a procedimiento de ablación de fibrilación auricular.

\section{Materiales y métodos}

\section{Pacientes}

Análisis retrospectivo de corte transversal, en el que se evaluaron pacientes a quienes se les hizo al menos un procedimiento de aislamiento eléctrico de venas pulmonares por fibrilación auricular paroxística, más seguimiento posterior mediante monitorización Holter entre octubre de 2010 y noviembre de 2014, en un centro de referencia de Medellín. El procedimiento se llevó a cabo mediante ablación con radiofrecuencia con catéter irrigado y aislamiento antral, según la técnica desarrollada por Natale et al. y Verma A, et $\mathrm{al}^{12}$.

Se seleccionaron aquellos cuya monitorización Holter a las cuatro semanas o hasta el tercer mes, evidenciara un incremento de la extrasistolia ventricular definido como tres o más CVPS por minuto o la presencia de TVNS. Igualmente, el grupo control lo conformaron aquellos en quienes la monitorización Holter durante el mismo periodo no mostró complejos ventriculares prematuros o TVNS.

Se excluyeron aquellos con marcapasos, arritmias diferentes a las ventriculares en la monitorización Holter y en quienes no fue posible el análisis de la variabilidad de la frecuencia cardiaca.

\section{Materiales}

Se utilizó el monitor Holter DMS $3003 \mathrm{~A}^{\circledR}$. La duración mínima de obtención de datos fue de 16 horas. Los datos se transfirieron al software CardioScan Premier ${ }^{\circledR}$ versión 12.2 de DMS para Windows, para el análisis inicial. La información de la variabilidad de la frecuencia cardiaca se obtuvo de los informes de medición de dominio tiempo y dominio frecuencia.

\section{Análisis de variabilidad de la frecuencia cardiaca}

La variabilidad de la frecuencia cardiaca se usó para representar la actividad cardiaca autonómica. Las densidades de espectro de poder se analizaron de acuerdo con el informe de la monitorización Holter. El componente de alta frecuencia (HF) $(0,15-0,4 \mathrm{~Hz})$ se empleó como un índice de la actividad parasimpática, en tanto que el de baja frecuencia (LF) $(0,05-0,15 \mathrm{~Hz})$ y su relación con el de HF (relación $\mathrm{LF} / \mathrm{HF}$ ) se usó como un índice de actividad simpática.
Tabla 1 Valores normales de la variabilidad de la frecuencia cardiaca

\begin{tabular}{lll}
\hline Parámetro & Valor normal & Unidad medida \\
\hline SDNN & $141 \pm 39$ & $\mathrm{~ms}$ \\
Poder total & $3466 \pm 1018$ & $\mathrm{~ms}^{2}$ \\
LF & $1170 \pm 416$ & $\mathrm{~ms}^{2}$ \\
HF & $975 \pm 203$ & $\mathrm{~ms}^{2}$ \\
Relación LF/HF & $1,5-2,0$ & - \\
\hline
\end{tabular}

Los valores de normalidad se basan en las guías acerca de la variabilidad de la frecuencia cardiaca publicadas por la Sociedad Europea de Cardiología y la Sociedad Norteamericana de Electrofisiología y marcapasos en $1996^{13}$ (tabla 1).

Valores de SDNN por debajo de 100 ms se consideraron como disminución leve de la variabilidad de la frecuencia cardiaca, mientras valores por debajo de $60 \mathrm{~ms}$ se consideraron compromiso severo. Valores de HF por debajo de lo normal se consideraron como disminución de la actividad parasimpática, mientras que valores por encima, como aumento en su actividad.

Así mismo, valores de LF por debajo de lo normal se consideraron como disminución de la actividad simpática, mientras que valores por encima como aumento en su actividad.

Valores de la relación LF/HF por debajo de 1,5 se consideraron como dominancia parasimpática, mientras que valores por encima de 2,0 se valoraron como cambio hacia la dominancia simpática.

\section{Análisis estadístico}

La información recolectada se organizó, examinó y estandarizó en un archivo de Microsoft Excel y se transfirió a IBM SPSS versión 20 para Mac, para su procesamiento respectivo. Las variables cualitativas se presentaron en número y porcentaje, y las cuantitativas como media y desviación estándar. Las diferencias entre los grupos de evaluación se valoraron con la prueba de Mann-Whitney para las variables cuantitativas y con el test de $\mathrm{chi}^{2}$ para las cualitativas.

\section{Aspectos éticos}

El estudio contó con la aprobación del Comité de Ética e Investigación Biomédica de la Universidad CES, Medellín, Colombia.

\section{Resultados}

\section{Pacientes}

Se hicieron en total 5.384 monitorizaciones Holter durante el 11 de octubre de 2010 y el 13 de noviembre de 2014, de las cuales $330(6,12 \%)$ correspondían a seguimientos de control postablación de fibrilación auricular, en 161 pacientes. Un total de 20 pacientes $(12,4 \%)$ tuvieron un incremento significativo del automatismo ventricular, con al menos tres CVPS por minuto o TVNS, demostrado por la monitorización Holter 
Tabla 2 Características basales de los pacientes

\begin{tabular}{|c|c|c|c|}
\hline & CVPS $(n=20)$ & Control $(n=34)$ & Valor $p$ \\
\hline \multicolumn{4}{|l|}{ Demográficos } \\
\hline Edad (Años, media, $\pm \mathrm{DE}$ ) & $66 \pm 9,7$ & $62 \pm 12,6$ & 0,309 \\
\hline Sexo masculino No. (\%) & $16(80,0)$ & $19(55,9)$ & 0,143 \\
\hline Peso (kg, media, $\pm \mathrm{DE})$ & $79,2 \pm 11,0$ & $75,4 \pm 13,0$ & 0,214 \\
\hline Talla $(\mathrm{m}$, media,$\pm \mathrm{DE})$ & $1,71 \pm 0,09$ & $1,68 \pm 0,08$ & 0,343 \\
\hline Índice de masa corporal $\left(\mathrm{m}^{2}\right.$, media, $\left.\pm \mathrm{DE}\right) 27,1 \pm 3,6$ & $26,4 \pm 2,92$ & 0,756 & \\
\hline \multicolumn{4}{|l|}{ Características CVPS } \\
\hline Frecuencia cardiaca promedio (lpm, media, $\pm \mathrm{DE})$ & $75,0 \pm 9,1$ & $76,8 \pm 10,0$ & 0,777 \\
\hline CVP total promedio (No. $\pm \mathrm{DE}$ ) & $5363 \pm 6616$ & 0 & 0,000 \\
\hline CVP/hora promedio (No. $\pm \mathrm{DE}$ ) & $237 \pm 291$ & 0 & 0,000 \\
\hline Taquicardia ventricular (No. \%) & $13(65)$ & $0(0)$ & 0,000 \\
\hline \multicolumn{4}{|l|}{ Medicación durante Holter - No. (\%) } \\
\hline Beta-bloqueador & $9(45)$ & $19(55,9)$ & 0,395 \\
\hline Amiodarona & $0(0)$ & $2(5,9)$ & 0,517 \\
\hline Propafenona & $0(0)$ & $2(5,9)$ & 0,517 \\
\hline
\end{tabular}

al primer o tercer mes postprocedimiento; en 34 pacientes $(21,1 \%)$ no se evidenciaron CVPS o TVNS y estos fueron seleccionados como grupo control.

La mayor proporción correspondió al sexo masculino $(64,8 \%)$, con una edad promedio de 64 años e índice de masa corporal de 26,7. Los datos demográficos en el grupo de CVPS, en comparación con el grupo control, no demostraron diferencias estadísticamente significativas en los parámetros valorados (tabla 2).

La frecuencia cardiaca promedio para el grupo de CVPS fue de 75 latidos por minuto, mientras que para el grupo control de 76,8, sin diferencias estadísticamente significativas $(p=0,777)$.

En el grupo de aumento del automatismo ventricular, el promedio de CVPS durante las 24 horas fue de 5.363 con 237 por hora. El 65\% de los pacientes en este grupo, presentaron TVNS.

En relación con el uso de medicamentos durante la monitorización Holter, en el grupo de CVPS el 45\% de los pacientes estaba medicado con betabloqueadores comparado con un $55,9 \%$ del grupo control, sin diferencias estadísticamente significativas $(p=0,395)$. Menos del $6 \%$ de los pacientes recibían amiodarona o propafenona en el grupo control, mientras en el grupo de CVPS ninguno recibía este tipo de medicación (tabla 2).

La variabilidad de la frecuencia cardiaca medida en dominio tiempo por la desviación estándar del intervalo $\mathrm{R}-\mathrm{R}$ normal en 24 horas (SDNN), tuvo un promedio menor en los pacientes del grupo control comparado con el grupo de CVPS, 94,5 vs. 138,6 milisegundos, con significancia estadística $(p=0,000)$. En el grupo de CVPS solo un paciente manifestó una variabilidad de la frecuencia cardiaca levemente disminuida, mientras en el grupo control el $61,4 \%$ presentó una disminución leve y cerca de un $6 \%$ disminución severa ( $p=0,000$ y 0,276 respectivamente). Su correlación, con la medición del poder total desde el punto espectral, confirmó una disminución de los valores en el $50 \%$ de los pacientes del grupo CVPS vs. $82,4 \%$ del control, con diferencias significativas $(p=0,005)$.
La variabilidad de la frecuencia cardiaca medida por las diferentes frecuencias espectrales, evidenció una disminución en la LF (marcador simpático) para ambos grupos, con un valor menor para el grupo control $\left(661,4\right.$ vs. $267,2 \mathrm{~ms}^{2}$, $\mathrm{p}=0,000$ ). El $60 \%$ de los pacientes del grupo de CVPS tenía una LF por debajo del rango normal comparado con el $82,4 \%$ del grupo control, con un valor de $\mathrm{p}$ estadísticamente significativo $(0,019)$. Solo 2 pacientes en el grupo CVPS tenían un valor de LF mayor a $1586 \mathrm{~ms}^{2}$, mientras en el grupo control ninguno de los pacientes presentaba LF elevada.

En cuanto a la HF (marcador parasimpático), ambos grupos tuvieron una disminución marcada de los valores, siendo menores para el grupo control (238,1 vs. $104,6 \mathrm{~ms})$, con diferencia estadísticamente significativa $(p=0,001)$. Diecinueve de 20 pacientes del grupo CVPS y 32 de 34 del grupo control, tenían valores por debajo de lo normal, sin significancia estadística (tabla 2).

La relación LF/HF para ambos grupos fue elevada, sin diferencias significativas $(3,21$ vs. 3,38$)(p=0,612)$.

El $17,6 \%$ de los pacientes en el grupo control y ninguno del grupo de CVPS tuvo una relación disminuida $(<1,5)$. Dieciséis de 20 pacientes en el grupo de CVPS y 22 de 34 del grupo control evidenciaron incremento en la relación (> $2,0)$, sin diferencias significativas en ninguno de los dos casos (tabla 3).

\section{Discusión}

La fibrilación auricular afecta aproximadamente al 1\% de la población general, con tasas que van en aumento, pues para el 2050 se estima una prevalencia de más de 30 millones de pacientes en Estados Unidos y Europa ${ }^{4,14}$. Por la poca eficacia de la terapia farmacológica, cada vez es más frecuente el uso de técnicas como la ablación con catéter y la ablación de venas pulmonares en casos seleccionados, ya que han demostrado ser superiores en el mantenimiento del ritmo sinusal, en la mejoría de los síntomas y posiblemente en la disminución del riesgo tromboembólico ${ }^{14}$. 
Tabla 3 Características de la frecuencia cardiaca

\begin{tabular}{|c|c|c|c|}
\hline & CVPS $(n=20)$ & Control $(n=34)$ & Valor $p$ \\
\hline \multicolumn{4}{|l|}{ Variabilidad de la frecuencia cardiaca } \\
\hline SDNN 24 horas (ms, $\pm \mathrm{DE})$ & $138,6 \pm 33,4$ & $94,5 \pm 28,3$ & 0,000 \\
\hline SDNN 24 horas 61 a 100 (ms, No., \%) & $1(5,0)$ & $21(61,8)$ & 0,000 \\
\hline SDNN 24 horas $<60$ (ms, No., \%) & $0(0)$ & $3(8,8)$ & 0,276 \\
\hline \multicolumn{4}{|l|}{ Poder espectral de la frecuencia cardiaca } \\
\hline Poder total promedio $\left(\mathrm{ms}^{2}\right.$, media, $\left.\pm \mathrm{DE}\right)$ & $2820,2 \pm 1520,6$ & $1342,1 \pm 1372,0$ & 0,000 \\
\hline Poder total $<2448 \mathrm{~ms}^{2}$ (No. \%) & $10(50)$ & $28(82,4)$ & 0,005 \\
\hline LF promedio $\left(\mathrm{ms}^{2}\right.$, media, $\left.\pm \mathrm{DE}\right)$ & $661,4 \pm 499,2$ & $267,2 \pm 324,1$ & 0,000 \\
\hline $\mathrm{LF}<754 \mathrm{~ms}^{2}$ (No. \%) & $12(60)$ & $28(82,4)$ & 0,019 \\
\hline $\mathrm{LF}>1586 \mathrm{~ms}^{2}$ (No. \%) & $2(10,0)$ & $0(0)$ & 0,143 \\
\hline HF promedio ( $\mathrm{ms}^{2}$, media, $\left.\pm \mathrm{DE}\right)$ & $238,1 \pm 269,0$ & $104,6 \pm 138,4$ & 0,001 \\
\hline $\mathrm{HF}<772 \mathrm{~ms}^{2}$ (No. \%) & $19(95,0)$ & $34(100)$ & 0,385 \\
\hline $\mathrm{HF}>1178 \mathrm{~ms}^{2}$ (No. \%) & $1(5,0)$ & $0(0)$ & 0,385 \\
\hline Relación LF/HF promedio (No. media) & 3,21 & 3,38 & 0,612 \\
\hline Relación LF/HF <1,5 (No. \%) & $0(0)$ & $6(17,6)$ & 0,071 \\
\hline Relación LF/HF >2,0 (No. \%) & $16(80,0)$ & $22(64,7)$ & 0,524 \\
\hline
\end{tabular}

La fisiopatología en el desarrollo de la fibrilación auricular resulta compleja. Dentro de los mecanismos implicados, la activación del SNA desempeña un papel importante al inducir cambios significativos y heterogéneos sobre la electrofisiología atrial ${ }^{1,6,15}$. Ciertos tipos de fibrilación auricular, como aquella que aparece durante el ejercicio, parecieran estar mediados por el estímulo simpático, mientras que aquella que se presenta en pacientes jóvenes con corazón estructuralmente sano pareciera estar mediada por el sistema nervioso parasimpático ${ }^{1}$. Las bases potenciales para que el SNA promueva la fibrilación auricular se asocian con mecanismos focales, que actúan como disparadores en un sustrato susceptible o al mantener la arritmia al proveer disparos rápidos, o asociados a mecanismos por reentrada.

La ablación de los plexos ganglionares del sistema nervioso cardiaco intrínseco durante el procedimiento de aislamiento de venas pulmonares, confiere una mayor tasa de éxito en términos de recurrencia; sin embargo, los efectos colaterales consecuentes aún no son claros ${ }^{6}$. Las respuestas vagales pueden verse afectadas significativamente por su impacto sobre el plexo ganglionar endocárdico, con eliminación completa de éstas después de la ablación ${ }^{14}$, hecho que puede afectar la modulación del tono autonómico que igualmente está implicado en la génesis, el mantenimiento y la interrupción de otros trastornos del ritmo, como las arritmias ventriculares ${ }^{16}$. De esta manera, la alteración de los plexos ganglionares autonómicos durante el aislamiento de venas pulmonares, pudiera incrementar la frecuencia de CVPS y TVNS $^{5}$. En población no seleccionada remitida para este procedimiento, como parte del tratamiento para fibrilación auricular paroxística o persistente, Patel et al. evidenciaron una incidencia de nuevo desarrollo de CVPS de alrededor del $11 \%{ }^{5}$. En el estudio que aquí se publica, se obtuvo un resultado similar, donde el $12,4 \%$ de la población valorada tenía un aumento importante del automatismo ventricular, con más de 3 CVPS por hora o TVNS. Aunque se ha sugerido que esta alteración pudiera ayudar a identificar aquellos pacientes con ablación vagolítica más extensa, en quienes adicionalmente se ha encontrado un incremento en la frecuencia cardiaca basal, los hallazgos de este reporte muestran datos contrarios, con un promedio de frecuencia cardiaca sin diferencias estadísticamente significativas en el grupo de CVPS comparado con el control.

El incremento en la frecuencia de CVPS usualmente se ha asociado con factores de riesgo cardiovascular tradicionales como hipertensión arterial, dislipidemia, diabetes y enfermedad coronaria ${ }^{17}$. Aunque en pacientes con corazón estructuralmente sano se acepta que la presencia de extrasístoles ventriculares con carga baja no eleva el riesgo de eventos cardiovasculares, no ocurre lo mismo en población con corazón estructuralmente enfermo en quienes se puede generar un incremento en el riesgo de arritmias malignas y muerte ${ }^{7,18}$.

El efecto de la modulación autonómica en aquellas arritmias ventriculares idiopáticas, es decir CVPS o TVNS en ausencia de enfermedad cardiaca estructural o funcional, es controversial. Estudios sobre la variabilidad de la frecuencia cardiaca han evidenciado cómo previo al inicio de una TVNS hay un aumento en la actividad simpática y/o disminución del tono vagal ${ }^{16}$. Sin embargo, otros estudios han encontrado que el aumento del tono vagal también se asocia con el desarrollo de estas arritmias ${ }^{16}$. En una publicación reciente se encontró que el control autonómico desempeña diferentes papeles en el desarrollo de CVPS idiopáticas, que puede verse reflejado por la dependencia de la frecuencia cardiaca. Algunos mecanismos pueden estar facilitados por la activación simpática que mejora la automaticidad, pero también se encontraron mecanismos por activación vagal, y aunque su influencia no se comprende del todo, pareciera estar relacionada con la facilitación de las posdespolarizaciones tempranas. En otros casos, el mecanismo implicado para el desarrollo de las CVPS se asoció con la disminución del tono vagal ${ }^{16}$. Las mediciones de la variabilidad de la frecuencia cardiaca se han utilizado como test no invasivo para la valoración de la función del SNA. Estas se dividen en dos categorías amplias: mediciones de dominio tiempo, que se emplean usualmente en análisis de 24 horas, y mediciones de dominio frecuencia, que se usan en grabaciones cortas 
de 5 minutos o en grabaciones prolongadas de 24 horas $^{11,13}$. Dentro de los parámetros clínicos más útiles está la desviación estándar del intervalo R-R normal (SDNN), que es un marcador del poder total (variación) de la variabilidad de la frecuencia cardiaca y refleja todos los componentes a largo plazo responsables de dicha variabilidad en el periodo de grabación, que usualmente es de 24 horas, incluyendo el ritmo circadiano y la actividad física. Cuando su valor es menor de $100 \mathrm{~ms}$ se considera un índice de flujo simpático anormal ${ }^{11}$. Las alteraciones en este índice se usan para predecir mortalidad en pacientes con enfermedad cardiaca estructural, principalmente enfermedad coronaria cuando los valores son menores a $70 \mathrm{~ms}^{11,19-21}$.

Hsieh et al. en un grupo de 30 pacientes llevados a procedimiento de ablación de venas pulmonares, evidenciaron una disminución de la variabilidad de la frecuencia cardiaca respecto al valor basal en términos de parámetros de dominio tiempo, incluyendo SDNN. Estos resultados sugerían una disfunción autonómica posprocedimiento por dominancia simpática o disminución de la actividad parasimpática ${ }^{10}$. De manera llamativa, los resultados del SDNN en la población de estudio en nuestro reporte, evidenciaron un impacto más relevante en el grupo control en comparación con el grupo de CVPS en cuanto a la disminución en el valor de dicho parámetro. Incluso, cerca de un $6 \%$ de los pacientes del grupo control tenían un valor de SDNN menor de $70 \mathrm{~ms}$, lo que representa una disminución severa de la variabilidad de la frecuencia cardiaca, mientras en el grupo CVPS ninguno de los pacientes estuvo por debajo de este valor. Estos resultados fueron corroborados con parámetros de dominio frecuencia como el poder total, en el que más del $82 \%$ de los pacientes del grupo control tenía un valor inferior al de referencia, mientras en el grupo de CVPS, solo la mitad tenía un valor anormal. El análisis del SDNN y de la potencia total en la población de nuestro estudio, mostró un impacto del aislamiento de las venas pulmonares sobre la variabilidad de la frecuencia cardiaca, el cual sin embargo no parece explicar el aumento en la incidencia de CVPS o TVNS en dicha población.

El análisis dominio frecuencia de la variabilidad de la frecuencia cardiaca, distingue dos componentes espectrales principales: componente de alta frecuencia ${ }^{1}(0,15$ a $0,4 \mathrm{~Hz})$, considerado como marcador vagal, y componente de baja frecuencia $(0,04 \mathrm{a} 0,15 \mathrm{~Hz})$ considerado como marcador simpático ${ }^{22}$. La relación entre ambos componentes se ha utilizado para valorar la distribución fraccional entre los dos sistemas y es un marcador importante del balance simpático-vagal. Una reducción en esta relación es un signo claro de desbalance entre las interacciones simpáticas reflejas con cambios hacia la disminución del simpático, y en consecuencia predominancia vagal. Los métodos de frecuencia dominio se usan para estudiar las variaciones de la frecuencia cardiaca al desglosar su señal en los constituyentes (frecuencias) y cuantificar su intensidad relativa (poder $)^{11}$. En general, valores bajos de la variabilidad de la frecuencia cardiaca usualmente indican una dominancia simpática relativa, que puede deberse a actividad simpática alta y/o actividad parasimpática baja. Por otra parte, valores altos de la variabilidad indican un cambio del balance simpático/parasimpático hacia mayor actividad vagal ${ }^{11}$.

En los grupos analizados en el presente reporte se documentó una alteración considerable en los parámetros de dominio frecuencia. La LF, que se ha utilizado como marcador simpático, estuvo marcadamente disminuida en ambos grupos, con mayor proporción para el grupo control. De igual forma, la HF, utilizada como marcador parasimpático, mostró una disminución significativa en ambos grupos, siendo mayor también en el grupo control. Se encontraron resultados similares en la población del estudio de Hsieh et al. en la que en el seguimiento postablación, ambas frecuencias disminuyeron de manera significativa desde el punto de vista estadístico ${ }^{10}$.

Cuando se analizó la relación LF/HF como marcador del balance simpático-vagal, se encontró muy elevada tanto para el grupo de control como para el de CVPS, hecho que indica un desbalance hacia el predominio simpático.

Pese a que ambos constituyentes del SNA medidos en términos de LF y HF estaban disminuidos de manera significativa, se sugiere que el impacto vagolítico es mucho mayor, razón por la cual puede existir un predominio simpático postablación y explicaría los valores altos de la relación LF/HF.

En términos de valores absolutos promedio en las LF y $\mathrm{HF}$, se evidencia que, a pesar de la disminución de estos en ambos grupos bajo el valor de referencia, el impacto fue significativamente mayor en el grupo control, con diferencias estadísticas. El valor para la LF en el grupo control fue de 267,2 $\mathrm{ms}^{2}$, mientras para el grupo CVPS de 661,4 $\mathrm{ms}^{2}$. En cuanto al valor de la HF para el grupo control fue de 104,6 $\mathrm{ms}^{2}$, en tanto que para el CVPS de $238,1 \mathrm{~ms}^{2}$. De acuerdo con estos valores se puede sugerir que para la población de estudio, el impacto de la ablación de venas pulmonares sobre el sistema simpático y parasimpático fue mucho menor para el grupo CVPS que para el control, y que a pesar de los valores similares de la relación LF/HF, el grupo de CVPS tuvo una actividad simpática proporcionalmente mayor con un impacto vagolítico menos considerable que lo esperado y que lo que sugieren otros estudios. Estas diferencias simpático-vagales que generan disfunción autonómica postablación, pueden ser uno de los factores implicados en el desarrollo de CVPS frecuentes y TVNS, si bien se requieren estudios adicionales que prueben dicha hipótesis.

\section{Limitaciones}

Por tratarse de un estudio de análisis retrospectivo de corte transversal, este puede estar sujeto a sesgo de selección. Los grupos comparativos variaron ostensiblemente en el número de pacientes analizados, aunque las características basales fueron similares. Así mismo, el número bajo de pacientes limita su poder y la posibilidad de generalizar los resultados obtenidos.

El análisis de la variabilidad de la frecuencia cardiaca se hace con base en los latidos sinusales del paciente. En aquellos casos donde existen arritmias o extrasístoles de cualquier índole, pueden verse alterados los valores de variabilidad de la frecuencia cardiaca, principalmente aquellos derivados del análisis dominio tiempo, hecho que, de cierta forma, explicaría las diferencias en los análisis del SDNN con las frecuencias de potencia total, LF y HF.

La variabilidad de la frecuencia cardiaca por dominio frecuencia se analizó de acuerdo con valores tomados en 
periodos prolongados cercanos a 24 horas. Aunque dichas frecuencias fueron tomadas en periodos cortos de grabación (5 minutos) y permiten valorar con más exactitud la variabilidad de la frecuencia cardiaca, con los datos obtenidos igualmente puede hacerse una comparación adecuada.

La ausencia de datos de seguimiento en estos pacientes impide valorar el comportamiento de los parámetros del Holter analizados a largo plazo, así como la prevalencia real de CVPS frecuentes y TVNS en pacientes postablación de fibrilación auricular.

\section{Conclusiones}

La ablación de venas pulmonares como parte del tratamiento de la fibrilación auricular, puede desencadenar nuevos trastornos arrítmicos, como CVPS frecuentes o TVNS, hasta en el $12 \%$ de los pacientes sometidos a esta intervención. La alteración de los plexos ganglionares cardiacos durante el procedimiento puede traducirse en disfunción autonómica, que pudiera explicar la mayor frecuencia de CVPS en esta población.

Por parámetros medidos en Holter de 24 horas, el análisis del tono autonómico sugiere un impacto importante sobre los diferentes parámetros de la variabilidad de la frecuencia cardiaca, que incluyen SDNN, LF, HF y relación LF/HF, en toda la población estudio.

En aquellos pacientes con CVPS frecuentes o TVNS postablación, el impacto sobre el sistema parasimpático y simpático pareciera ser menor que en aquellos sin estas alteraciones, fenómeno que sugiere un desbalance autonómico de predominancia simpática, con menor impacto sobre la LF.

Si bien la disfunción autonómica puede ser un factor importante para el desarrollo de trastornos arrítmicos ventriculares en pacientes postablación de fibrilación auricular, es posible que no sea el único implicado en la fisiopatología de dicho trastorno.

En la literatura se dispone de poca información sobre el desarrollo de arritmias ventriculares en población intervenida para ablación de fibrilación auricular. Este estudio abre las puertas al adelanto de nuevas investigaciones sobre el papel del SNA en esta condición.

Se requieren estudios adicionales para valorar la incidencia real de esta alteración, su impacto a largo plazo, así como otros factores implicados.

\section{Responsabilidades éticas}

Protección de personas y animales. Los autores declaran que para esta investigación no se han realizado experimentos en seres humanos ni en animales.

Confidencialidad de los datos. Los autores declaran que han seguido los protocolos de su centro de trabajo sobre la publicación de datos de pacientes.

Derecho a la privacidad y consentimiento informado. Los autores han obtenido el consentimiento informado de los pacientes y/o sujetos referidos en el artículo. Este documento obra en poder del autor de correspondencia.

\section{Financiación}

Ninguna

\section{Conflicto de intereses}

Ninguno

\section{Bibliografía}

1. Linz D, Ukena C, Mahfoud F, Neuberger HR, Bohm M. Atrial autonomic innervation: a target for interventional antiarrhythmic therapy. J Am Coll Cardiol. 2014;63:215-24.

2. Woods CE, Olgin J. Atrial fibrillation therapy now and in the future: drugs, biologicals, and ablation. Circulation Research. 2014;114:1532-46.

3. Kumagai K. Catheter ablation of atrial fibrillation. -State of the art. Circ J. 2011;75:2305-11.

4. Haegeli LM, Calkins H. Catheter ablation of atrial fibrillation: an update. Eur Heart J. 2014;35:2454-9.

5. Patel PJ, Ahlemeyer L, Freas M, Cooper JM, Marchlinski FE, Callans DJ, et al. Outflow tract premature ventricular depolarizations after atrial fibrillation ablation may reflect autonomic influences. J Interv Card Electrophysiol. 2014;41:187-92.

6. Chen PS, Chen LS, Fishbein MC, Lin SF, Nattel S. Role of the autonomic nervous system in atrial fibrillation: pathophysiology and therapy. Circulation Research. 2014;114:1500-15.

7. Ng GA. Treating patients with ventricular ectopic beats. Heart. 2006;92:1707-12.

8. Adams JC, Srivathsan K, Shen WK. Advances in management of premature ventricular contractions. J Interv Card Electrophysiol. 2012;35:137-49.

9. Cantillon DJ. Evaluation and management of premature ventricular complexes. Cleveland Clin J Med. 2013;80:377-87.

10. Hsieh MH, Chiou CW, Wen ZC, Wu CH, Tai CT, Tsai CT, et al. Alterations of heart rate variability after radiofrequency catheter ablation of focal atrial fibrillation originating from pulmonary veins. Circulation. 1999;100:2237-43.

11. Xhyheri B, Manfrini O, Mazzolini M, Pizzi C, Bugiardini R. Heart rate variability today. Prog Cardiovasc Dis. 2012;55:321-31.

12. Verma A, Marrouche NF, Natale A. Pulmonary vein antrum isolation: intracardiac echocardiography-guided technique. J Cardiovasc Electrophysiol. 2004;15:1335-40.

13. Task Force of the European Society of Cardiology, the North American Society of Pacing, Electrophysiology. Heart rate variability. Standards of measurement, physiological interpretation, and clinical use. Eur Heart J. 1996;17:354-81.

14. Santangeli P, Di Biase L, Burkhardt DJ, Horton R, Sanchez J, Bai R, et al. Catheter ablation of atrial fibrillation: state-ofthe-art techniques and future perspectives. J Cardiovasc Med (Hagerstown). 2012;13:108-24.

15. Nishida K, Datino T, Macle L, Nattel S. Atrial fibrillation ablation: translating basic mechanistic insights to the patient. J Am Coll Cardiol. 2014;64:823-31.

16. He W, Lu Z, Bao M, Yu L, He B, Zhang Y, et al. Autonomic involvement in idiopathic premature ventricular contractions. Clin Res Cardiol. 2013;102:361-70.

17. Ephrem G, Levine M, Friedmann P, Schweitzer P. The prognostic significance of frequency and morphology of premature ventricular complexes during ambulatory holter monitoring. Ann Noninvasive Electrocardiol. 2013;18:118-25.

18. Lee YH, Zhong L, Roger VL, Asirvatham SJ, Shen WK, Slusser $\mathrm{JP}$, et al. Frequency, origin, and outcome of ventricular premature complexes in patients with or without heart diseases. Am J Cardiol. 2014;114:1373-8. 
19. Huikuri HV, Stein PK. Heart rate variability in risk stratification of cardiac patients. Prog Cardiovasc Dis. 2013;56:153-9.

20. Kudaiberdieva G, Gorenek B, Timuralp B. Heart rate variability as a predictor of sudden cardiac death. Anadolu Kardiyol Derg. 2007;7 Suppl 1:68-70.
21. Bilchick KC, Berger RD. Heart rate variability. J Cardiovasc Electrophysiol. 2006;17:691-4.

22. Billman GE. The LF/HF ratio does not accurately measure cardiac sympatho-vagal balance. Front Physiol. 2013;4: 26. 